\title{
Vivencias de padres de niños con fisura labio palatina respecto al cuidado en las distintas etapas del tratamiento quirúrgico
}

\section{Experiences of parents of children with cleft lip palate regarding care in the different stages of surgical treatment}

\section{Vivências de pais de crianças com fissura labiopalatina quanto aos cuidados nas diferentes etapas do tratamento cirúrgico}

\author{
Verónica San Martín Osses ${ }^{1}$, ORCID 0000-0002-9450-946X \\ Ximena Osorio Spuler ${ }^{2}$, ORCID 0000-0003-1885-8016 \\ ${ }^{1}$ Universidad Mayor. Chile \\ ${ }^{2}$ Universidad de La Frontera. Chile
}

Recibido: 28/06/2020

Aceptado: 21/04/2021

\begin{abstract}
Resumen: La enfermera(o) es uno de los profesionales de la salud encargado(a) de proporcionar cuidados al paciente con malformación labio palatina durante la evolución de su tratamiento. Sin embargo, no existen suficientes estudios nacionales ni internacionales que evidencian la vivencia de los padres en relación al cuidado recibido por sus hijos, debido a lo cual nace la inquietud de investigar la experiencia de un grupo de padres del sur de Chile respecto a los cuidados recibidos por parte del profesional enfermero durante la evolución de la enfermedad de sus hijos. El objetivo es develar vivencias de padres de niños con malformación labio palatina, con respecto a los cuidados de enfermería recibidos desde que el niño es diagnosticado hasta que es sometido al procedimiento quirúrgico, en el hospital Hernán Henríquez Aravena, durante el primer semestre de 2019. Es un estudio cualitativo con enfoque fenomenológico descriptivo, realizado a través de entrevistas en profundidad, notas de campo y grabaciones de audio. Respecto de los resultados, de las vivencias y narraciones de los padres surgen categorías y subcategorías que permiten analizar más claramente la investigación. Estas categorías son: emociones negativas vividas, experiencia con los cuidados en salud y expectativas de cuidados de enfermería. Se concluye que los padres perciben una permanente invisibilidad de enfermería, confundiendo muchas veces su atención con la proporcionada por técnicos y/u otros profesionales de la salud. En cuanto a los cuidados de enfermería, relatan gratitud, pero sin distinción de quién la propició, sino más bien hacia el equipo de salud en general.
\end{abstract}

Palabras clave: fisura del paladar; ansiedad; enfermería; cuidados de enfermería; emociones. 


\begin{abstract}
The nurse is one of the healthcare professionals in charge of providing care to the patient with cleft lip palate malformation during the evolution of the treatment. However, there are not enough national or international studies regarding the parents' experience with the care that their children received, due to which the concern arises to investigate the experience of a group of parents from the south of Chile regarding the care that their children received from a professional nurse during the evolution of their child's disease. The objective is to unveil the experience of the parents of children with cleft lip palate regarding the nursing care received since the child is diagnosed until he/she is submitted to the surgical treatment, in the Hernan Henríquez Aravena Hospital during the first semester of 2019. It is a qualitative study with a descriptive phenomenological approach, done through an in-deep interview, field notes and audio recordings. Regarding the results, from the experience and the narrations of the parents, categories and subcategories arise allowing analyzing more clearly the investigation. These categories are: negative emotions experienced, experience with wealth care and expectations from nursing care. In conclusion the parents receive a permanent nursing invisibility, often confusing their care with the one provided by technicians and/or other healthcare professionals. Regarding nursing care, they express gratitude, but without distinction of who provided it, just to the healthcare team in general.
\end{abstract}

Keywords: cleft palate; anxiety; nursing; nursing care; emotions.

Resumo: O enfermeiro é um dos profissionais de saúde encarregados de cuidar do paciente com malformação labiopalatina durante a evolução do seu tratamento. No entanto, não existem estudos nacionais ou internacionais suficientes que mostrem a experiência dos pais em relação ao cuidado recebido por seus filhos, por isso surge a preocupação em investigar a experiência de um grupo de pais no sul do Chile a respeito dos cuidados recebidos pela enfermagem. Profissional durante a evolução da doença dos filhos. O objetivo é revelar experiências de pais de crianças com malformação labiopalatina, a respeito dos cuidados de enfermagem recebidos desde o momento do diagnóstico da criança até a realização do procedimento cirúrgico, no hospital Hernán Aravena, durante o primeiro semestre de 2019. É um estudo qualitativo com abordagem fenomenológica descritiva, realizado por meio de entrevistas em profundidade, notas de campo e gravações em áudio. Em relação aos resultados, emergem as vivências e narrativas dos pais, categorias e subcategorias que permitem uma análise mais clara da pesquisa. Essas categorias são: emoções negativas vivenciadas, vivência com o cuidado à saúde e expectativas do cuidado de enfermagem. Conclui-se que os pais percebem uma invisibilidade permanente da enfermagem, muitas vezes confundido seus cuidados com os prestados por técnicos e/ ou outros profissionais de saúde. Quanto aos cuidados de enfermagem, relatam gratidão, mas sem distinção de quem os fomentou, mas sim para com a equipe de saúde em geral.

Palavras-chave: fissura palatina; ansiedade; enfermagem; cuidados de enfermagem; emoções.

Correspondencia: Verónica San Martín Osses, e-mail: veronicasanm@gmail.com 


\section{Introducción}

El embarazo es un momento de gran expectativa para los padres, quienes suelen idealizar al futuro hijo, y solo después del nacimiento, la brecha entre el recién nacido imaginario y el real se deshace. De esta forma, cuando ocurre un suceso imprevisto, como el nacimiento de un niño con alguna malformación, los padres experimentan un brote de sentimientos inesperados (1). Cuando corresponde a una malformación labio palatina, suele ser estresante debido a la gran cantidad de problemas infantiles que surgen producto de la enfermedad, como la alimentación, el aspecto facial y la vocalización, entre otros. Los padres comentan que hacer frente al diagnóstico, reorganizar la estructura familiar y someterse al tratamiento y hospitalización puede ser difícil (2, $3)$.

En cuanto a la fisura labio palatina, la incidencia en Chile corresponde a 1.7 por 1000 recién nacidos, alrededor de 400 casos al año; y en la región de la Araucanía, de acuerdo con la información proporcionada por una enfermera de la Unidad del Niño Fisurado del hospital Hernán Henríquez Aravena (HHHA), entre 2005 y 2018 nacieron 605 niños con esta malformación. Entre su etiología se describen factores hereditarios, ambientales y desconocidos. En cuanto al tratamiento, es principalmente quirúrgico, interviniendo un equipo multidisciplinario. Inicia entre los 3 a 6 meses de vida y continúa hasta los 15 años, con distintas cirugías según la edad del paciente $(4,5)$. En relación al proceso quirúrgico, Gómez-Carretero describe que en la etapa preoperatoria surgen sentimientos como la ansiedad (6); además, en el caso del niño, una cirugía es una fuente de estrés psicológico, debido al enfrentamiento a una situación desconocida, sumándose los sentimientos de temor de sus padres frente a este acontecimiento (7). Cuando los padres experimentan este tipo de sentimientos sin lograr su control, podrían generar emociones de inseguridad en el niño, pudiendo deberse a falta de información sobre el proceso quirúrgico, más que a la cirugía en sí (8). Por esto, la importancia de la información preoperatoria, que mejora la comprensión sobre el proceso, reduciendo sentimientos de ansiedad y estrés frente a la intervención $(9,10)$.

Por otro lado, es importante que el medio hospitalario ofrezca las condiciones necesarias para afrontar el estrés del tratamiento, cobrando importancia los cuidados de enfermería, debido a que son, quienes están más tiempo con los pacientes y su familia, ayudando en este proceso de adaptación, a través de un comportamiento cálido y empático $(6,9)$. Es por esto que conocer cómo percibe el familiar acompañante el cuidado del equipo de enfermería es una herramienta para encontrar soluciones y mejorar la calidad del servicio. Esto es uno de los retos principales, que tanto el paciente como su familia manifiesten confianza y seguridad en el cuidado proporcionado por las(os) enfermeras(os); este cuidado será percibido en la respuesta que presenten ambos, el reflejo objetivado de la satisfacción como usuarios, por ello es importante ser rigurosos y delicados en su entrega, para asegurar cuidados de calidad (11).

En lo que respecta a las políticas públicas de salud en Chile, el documento "Estrategia Nacional de Salud para el cumplimiento de los Objetivos Sanitarios de la Década 2011-2020", plantea que tanto las metas como indicadores expuestos, responden a una rigurosa priorización de temas sanitarios, centrándose prioritariamente en el análisis y elaboración de propuestas para los diferentes ámbitos relacionados con la salud, los cuales se enfocan en nueve áreas temáticas y cuatro objetivos generales (12). La presente investigación aborda el tema de satisfacción usuaria, que corresponde al resultado de la provisión de servicios de acuerdo a las necesidades, siendo una preocupación permanente de los servicios de salud (12). 
A raíz de lo antes expuesto, surge la inquietud de investigar sobre las vivencias de madres y/o padres de niños con malformación labio palatina respecto a los cuidados de enfermería recibidos durante el proceso quirúrgico. Teniendo en consideración también el alto porcentaje de recién nacidos con este tipo de malformación en el país, la falta de estudios publicados sobre las vivencias de los padres respecto a los cuidados de enfermería, siendo estos últimos quienes proporcionan atención directa durante el transcurso del tratamiento. Por otra parte, la utilidad y el valor social de la investigación están en describir el fenómeno desde la perspectiva de los padres, los cuales han sido clasificados en función a una patología específica y su evolución en el tiempo.

\section{Objetivo}

Develar vivencias de padres de niños con malformación labio palatina, con respecto a los cuidados de enfermería recibidos, desde que el niño es diagnosticado hasta que es sometido al proceso quirúrgico, en el hospital Hernán Henríquez Aravena, durante el primer semestre de 2019.

\section{Metodología}

Este es un estudio cualitativo con enfoque fenomenológico descriptivo; una vez recolectada la información se realizó un análisis con carácter netamente subjetivo, permitiendo un acercamiento a las experiencias del grupo en estudio (13-15), rescatando la esencia de cada entrevista respecto a los cuidados de enfermería que recibieron en un período determinado. Como referente metodológico filosófico, la investigación se apoya en la teoría de Edmund Husserl (1620), y como referente de enfermería en la teoría de los cuidados de Kristen Swanson (21, 22).

La población de estudio son padres de niños con malformación de fisura labio palatina, que se encuentren en la etapa del diagnóstico o ya han sido sometidos al proceso quirúrgico, que es parte del tratamiento de la enfermedad. La muestra se obtuvo de la base de datos de enfermería, perteneciente a la Unidad de Niños Fisurados, del HHHA en 2019, previa autorización del director de la institución y del Comité Ético Científico del Servicio Araucanía Sur. Una vez recopilada la información, se ubicó a los padres que asistieron a control médico y que cumplían con los criterios de inclusión, invitándolos a participar en el proyecto, posteriormente se realizó una entrevista en profundidad a cada participante. La población estudiada corresponde al total que permitió la saturación de información respecto a los objetivos planteados. En cuanto al tipo de muestra, pertenece a una muestra homogénea combinada con casos-tipo, ya que la población de estudio posee como característica similar el diagnóstico médico de sus hijos, rescatando además la esencia de las narraciones (23). Los criterios de inclusión fueron: madres y/o padres de niños atendidos en el HHHA debido a su fisura labio palatina.

La recolección de datos se obtuvo a través de entrevistas en profundidad, notas de campo y grabaciones de audio. Posteriormente, fueron transcritos y analizados hasta la saturación de la información, criterio definitorio para detener el proceso de selección de informantes (13).

Respecto al plan de análisis, se realizó de manera manual e informática, esta última con apoyo del programa Atlas-ti versión 8,4. De esta forma se redujeron los datos, hasta lograr la categorización, codificación, síntesis y agrupamiento de estos $(13,24)$. Finalmente, se obtuvieron los resultados y conclusiones, permitiendo comprender y conocer la realidad estudiada (23).

En relación con las consideraciones éticas, además de la evaluación del Comité Ético Científico del Servicio Araucanía Sur y la autorización del HHHA, se aplicaron los principios éticos de E. Emanuel (25); aplicando un consentimiento informado a los participantes, quienes 
fueron tratados con respeto, manteniendo la privacidad y resguardando la información entregada. Se les asignó un seudónimo para facilitar el análisis de los relatos. En cuanto a los criterios de rigor, para salvaguardar la calidad científica del estudio, se utilizaron los criterios descritos por Guba y Lincoln (26).

\section{Resultados}

Se develó la vivencia de los padres durante las distintas etapas de la vida en que sus hijos fueron hospitalizados y cuidados por enfermeras(os) para su recuperación, surgiendo de sus narraciones categorías y subcategorías que son analizadas a continuación:

Tabla 1: Descripción de las categorías y subcategorías, de acuerdo a los objetivos planteados y a lo señalado por los entrevistados.

\begin{tabular}{|c|c|c|}
\hline Categoría & Subcategoría & Definición de categoría \\
\hline $\begin{array}{l}\text { 1.Emociones } \\
\text { negativas vividas }\end{array}$ & $\begin{array}{l}\text {-Impresión después de la } \\
\text { cirugía } \\
\text {-Diagnóstico difícil de } \\
\text { enfrentar } \\
\text {-Experiencia traumática }\end{array}$ & $\begin{array}{l}\text { Impresiones experimentadas por los } \\
\text { entrevistados, comienzan cuando se } \\
\text { enteran de la malformación de sus hijos, } \\
\text { evolucionando en el transcurso del } \\
\text { proceso, por ejemplo, madres } \\
\text { imposibilitadas de amamantar al recién } \\
\text { nacido, enfrentan solas el tratamiento; } \\
\text { padres que sumen rol de proveedor no } \\
\text { pueden acompañar a sus hijos. }\end{array}$ \\
\hline $\begin{array}{l}\text { 2.Experiencia con } \\
\text { los cuidados en } \\
\text { salud }\end{array}$ & $\begin{array}{l}\text { - Invisibilidad de enfermería } \\
\text { - Rol de enfermería } \\
\text { - Reconociendo a otros } \\
\text { integrantes del equipo de salud }\end{array}$ & $\begin{array}{lll}\text { Los cuidados y las subcategorías, se } \\
\text { relacionan principalmente con la } \\
\text { hospitalización en la unidad de } \\
\text { recuperación anestésica y el servicio de } \\
\text { cirugía pediátrica. }\end{array}$ \\
\hline $\begin{array}{l}\text { 3.Expectativas de } \\
\text { cuidados de } \\
\text { enfermería }\end{array}$ & & $\begin{array}{l}\text { Requerimientos del cuidado de enfermería } \\
\text { esperados por los padres durante el } \\
\text { tratamiento de sus hijos. }\end{array}$ \\
\hline
\end{tabular}


Figura 1: Familia de categorías y subcategorías, de acuerdo a los relatos de los entrevistados. Análisis programa Atlas-Ti.

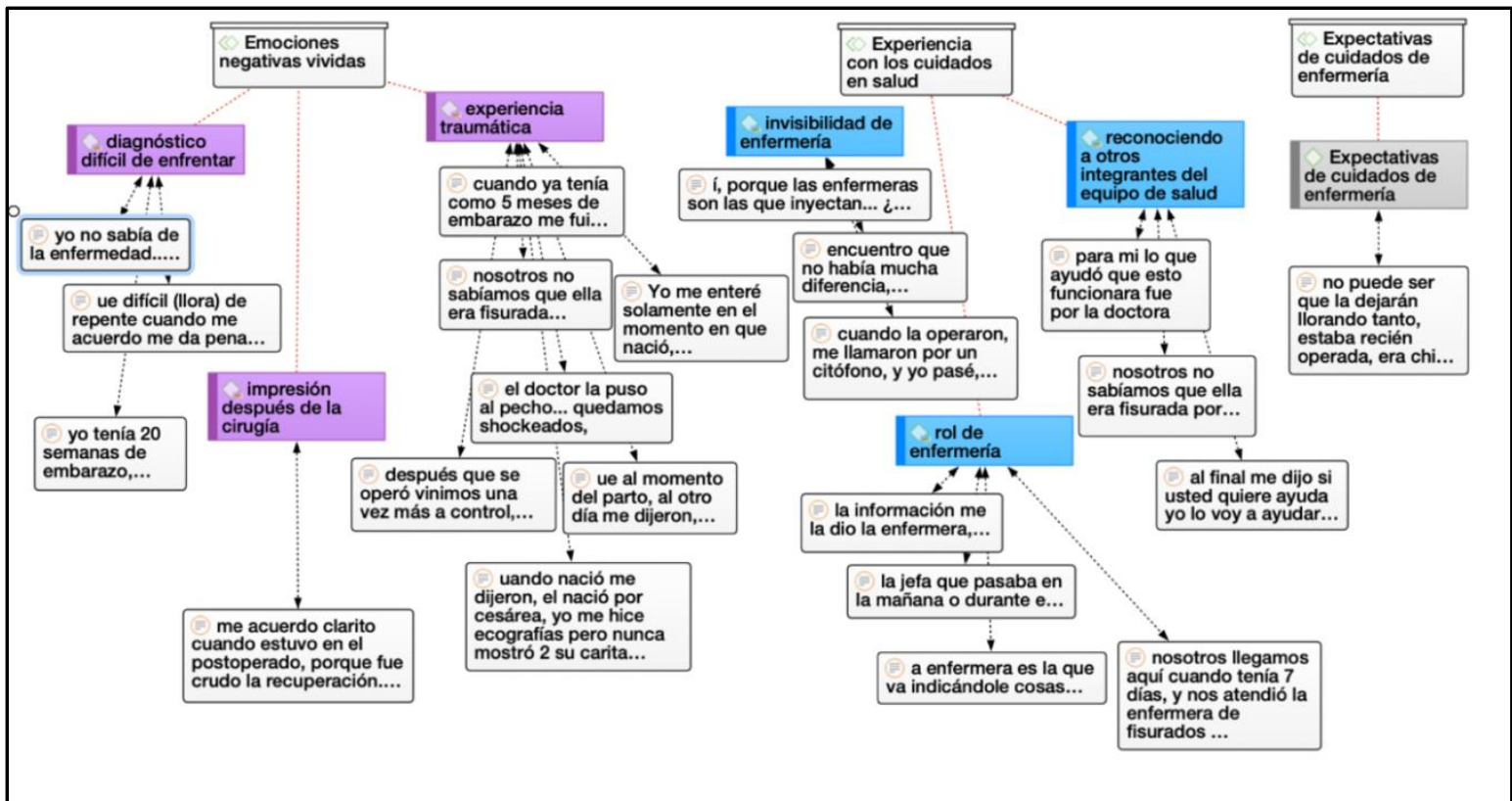

A continuación se analiza cada categoría:

\section{Emociones negativas vividas}

Esta dimensión está presente en la mayoría de los entrevistados, de allí se desprenden tres subcategorías: impresión después de la cirugía, diagnóstico difícil de enfrentar y experiencia traumática, que se apoyan en los siguientes relatos:

Impresión después de la cirugía, condición del niño al salir de pabellón: "Cuando estuvo en el postoperatorio fue cruda la recuperación, su boca venía con una prótesis plástica, abierta y llena de sangre, se quejó mucho de dolor, fue impresionante" (E1).

Diagnóstico difícil de enfrentar, padres que se enteran de la malformación: "No sabía de la enfermedad, cuando me hicieron la ecografía dijeron: "Viene bien la bebé, pero con una malformación», fue difícil" (E5). "Fue difícil, cuando me acuerdo me da pena, yo lloraba, no sabía qué hacer, me decían que no tenía que estar triste, que había tratamiento si ella sobrevivía” (E7). El llanto de las madres al momento de ambas entrevistas sustenta el relato (NC 5, 7). "Tenía 20 semanas de embarazo, la ecografía mostró la malformación, fue complicado porque uno siempre piensa que los hijos van a venir bien" (E9). 
Experiencia traumática, vivencias frente al diagnóstico inesperado, refirieron falta de información sobre la patología y sus eventuales complicaciones. Las madres, imposibilitadas para amamantar, se sintieron solas durante el tratamiento, debido a que los padres tuvieron que seguir trabajando:

Cuando nació me dijeron que tenía esta enfermedad, fue fuerte, me puse a llorar, no entendía por qué, estaba tan ilusionada de tener una hija. Le costaba succionar, tuvieron que alimentarla con la otra leche (E2).

Me enteré cuando nació, no entendía nada, cuando quise amamantarla no pude, fue terrible. No podía succionar porque se ahogaba, así que me tuve que sacar leche y dársela con cuchara, después que la operaron me quedé día y noche con ella, fue cansador (E4).

Mi hija nació con fisura palatina, me enteré al otro día, pero no entendí nada, no succionaba. Después que se operó vinimos solo una vez a control, me aburría de venir al hospital, a veces tenía que traerla sin dormir toda la noche, era agotador, venía sola porque mi esposo trabajaba (E6).

\section{Experiencia con los cuidados en salud}

Estas experiencias están marcadas por las relaciones establecidas con los distintos integrantes del equipo de la salud. De los relatos surgen tres subcategorías: invisibilidad de enfermería, rol de enfermería y reconociendo a otros integrantes del equipo de la salud. A continuación se citan los relatos:

Invisibilidad de enfermería, poca claridad percibida por los padres respecto a los cuidados de enfermería, no logran distinguir a la(el) enfermera(o) del resto del equipo, refieren identificarlos solo por la ejecución de procedimientos específicos o por su uniforme azul:

No había mucha diferencia entre quienes trabajaban en la sala de recuperación, nadie se presentó, pienso que la señorita que me fue a buscar para acompañar a mi hija era enfermera, no sé en realidad, por el uniforme azul se pueden distinguir (E2).

Cuando la operaron, me llamaron por un citófono, me dijeron "Su guagüita se está despertando", era una sala redonda, a la enfermera le podíamos hacer consultas solo si habían dudas, ella no se acercó donde nosotros, estaba con los pacientes complicados (E3).

En la sala de recuperación no se diferenciaba quién era enfermera o quien era técnico, en general, mi hija no se ha atendido mucho con enfermeras, son los técnicos quienes han estado con ella (E4).

Rol de enfermería, lo relacionan principalmente con funciones administrativas, como jefatura, supervisión del personal e indicación de procedimientos:

La información me la dio la enfermera, todo lo del cuidado, la enfermera del CDT se encargaba más de lo administrativo (E1). 
La enfermera es la jefa, pasaba en la mañana o durante el día, supervisando la ficha, miraba un poco y preguntaba algunas cosas, eso no más (E2).

Llegamos aquí cuando mi hija tenía 7 días, nos atendió la enfermera del programa de fisurados, ella nos entregó toda la información, siempre estuvo dispuesta a ayudarnos (E3).

La enfermera es quien va indicándole cosas al resto, cuando llega la otra enfermera a recibir el turno se presentan y luego se entregan, ahí nosotros vamos conociendo a quien le va a tocar estar de turno (E7).

Reconociendo a otros integrantes del equipo de la salud, relacionado con la atención otorgada por otros miembros del equipo, distintos a la(el) enfermera(o), destacan médicos cirujanos y técnicos en enfermería:

Para mí lo que ayudó para que esto funcionara fue la doctora, cuando nació nos dimos cuenta, me comuniqué con la doctora y me dijo: "Si usted quiere ayuda yo lo voy a ayudar", nos exigió que no podíamos faltar a ningún control, para nosotros ella fue quién nos abrió las puertas. Cuando uno es profesional cree que nunca va a necesitar la ayuda de otra persona, pero cuando nació mi hija, hasta la persona que barría el piso me servía, porque yo andaba perdido, ahí me di cuenta de que cada persona vale, que necesitamos de todos (E3).

Cuando nació me dijeron: "Tienes que irte a Temuco con la doctora encargada del programa de fisurados", ella nos preguntó si tomábamos o dejábamos el tratamiento, mi esposo dijo vamos a tomar el tratamiento, seguimos haciendo todo lo que nos decían (E7).

\section{Expectativas de cuidados de enfermería}

En lo concerniente con los requerimientos del cuidado de enfermería, lo que ellos hubiesen esperado durante la hospitalización de sus hijos, se destaca en sus relatos la esperanza de obtener mayor presencia y cercanía por parte de las(os) enfermeras(os):

No puede ser que la dejaran llorando tanto, estaba recién operada, siento que no se preocuparon mucho de ella. Hubiese querido estar desde el principio en la sala de recuperación, que trataran de calmar a la niña, necesitamos más apoyo, más información. Después estuvo hospitalizada en cirugía infantil, estuve con ella todo el tiempo, pero no me gustó que solo las mamás se podían quedar en la noche, uno igual necesita descansar, ahí la enfermera era la jefa, pasaba en la mañana o durante el día, supervisando la ficha, miraba un poco y preguntaba algunas cosas, eso no más (E2).

Ahí estaba la enfermera, cualquier cosa uno le podía consultar, pero solo si teníamos dudas, ella no se acercó donde nosotros (E3). 


\section{Discusión y consideraciones finales}

Los resultados evidenciaron cierta similitud en los relatos, destacando la forma de enfrentar el diagnóstico, muchas veces inesperado; surgiendo sentimientos y emociones negativas, como dolor, tristeza e incertidumbre. Por otra parte, se desvela la percepción de los padres respecto a los cuidados de enfermería, en los distintos momentos que fueron requeridos por sus hijos; identificando, además, el rol de la enfermera(o) de acuerdo al concepto que tienen de ella(el), lo cual es contrastado con la invisibilidad de enfermería, reconociendo lo difícil que fue distinguir a la(el) enfermera(o) del resto de los funcionarios que los atendieron.

El nacimiento de un hijo con una malformación suele ser una situación inesperada, generando gran impacto, aflorando sentimientos de angustia, negación y confusión, que se presentan de forma consciente o inconsciente, y se expresan como una experiencia traumática, llevando a un estado de vulnerabilidad $(5,27,28)$; estos sentimientos fueron narradas por los padres entrevistados, respaldando los resultados de la investigación. Respecto a la enfermedad de un niño, esta suele afectar tanto al niño como a sus padres, reaccionando estos últimos de diferentes maneras. Las madres suelen presentar sentimientos de tristeza y angustia, siendo ellas en la mayoría de los casos quienes se encargan de su cuidado, tal como se demostró en el estudio "Madres cuidadoras de niños(as) con cáncer: vivencias, percepciones y necesidades" realizado por Lafaurie et al (29); y esto se debe a que la enfermedad implica una reorganización familiar, donde los padres deben tomar decisiones como quién asumirá el rol de cuidador primario. Esto fue evidenciado en la investigación realizada por Grau et al, "Percepciones de los padres de niños enfermos de cáncer sobre los cambios en las relaciones familiares" (30). De igual forma el rol de "madre cuidadora" se presentó en algunas de las madres estudiadas, quienes justificaron la ausencia del padre, porque ellos debieron seguir proveyendo a la familia. Por otro lado, complementando la experiencia traumática experimentada por ellas, la lactancia materna fue otro factor que acrecentó este estado, ya que las alteraciones anatómicas y funcionales del recién nacido dificultan su alimentación, ocasionando sentimiento de pérdida en las madres, quienes suelen tener el deseo de amamantarlo, interfiriendo en el vínculo madre-hijo, influenciado además por el estado emocional de ellas, las expectativas previas de este proceso y la sensación de incompetencia. Esto fue evidenciado en estudios como "Guía Clínica AUGE Fisura Labiopalatina" del Ministerio de Salud, "Rigor y calidad metodológicos: un reto a la investigación social cualitativa" de Cornejo y "Madres cuidadoras de niños(as) con cáncer: vivencias, percepciones y necesidades" de Lafaurie et al $(5,26,29)$, presentándose en la mayoría de las madres, quienes al recordar la experiencia se pusieron a llorar. Las reacciones antes mencionadas se revelaron en ambos padres, principalmente cuando se enteran del estado de sus hijos, ellos describieron sentimientos negativos, señalando que fue una situación difícil y complicada de enfrentar; similar al estudio de Badillo et al (28). De esta forma, las investigaciones expuestas respaldan el presente estudio, evidenciando similitudes en la mayoría de las vivencias narradas.

Continuando con el orden de las categorías, se hace mención a que la salud y enfermedad son circunstancias habituales en las personas, y cada grupo social desarrolla distintas estrategias en salud para darles solución, de esta forma, el cuidado en salud se entiende como una práctica social. Es así como, el cuidado de enfermería nace de esta génesis común con el cuidado de la salud (31), en este contexto el cuidado de enfermería comprende un compromiso humanista e interés por sus semejantes y su entorno, características de la gestión del cuidado, la cual se entiende como el ejercicio profesional de enfermería, apoyada en su disciplina, la ciencia de cuidar. La gestión del cuidado debe permitir brindar un cuidado planificado, estableciendo objetivos, metas 
y estrategias, que implican la creatividad del profesional, asegurando la calidad del cuidado que se otorga a los usuarios y a su familia. Para lograr esto, la(el) enfermera(o) debe ver al individuo como un ser holístico, incluyendo una mirada biológica, escucha activa, respetando en todo momento sus derechos, tal como se revela en los estudios realizados por Milos, Grau y Ortiz (3234); sin embargo, contrastando la definición de cuidado de enfermería, la presente investigación revela una(o) enfermera(o) distante, poco empática, más preocupada de las tareas administrativas que del acompañamiento.

De acuerdo a lo investigado por Suárez et al (35), en un grupo de madres de niños que padecían algún tipo de malformación congénita, refirió que las madres sintieron discriminación hacia sus hijos, lo cual era esperable si venía de la sociedad, pero no cuando se presentaba en el equipo de salud, provocando un impacto negativo e incomprensible en ellas; esta discriminación fue presentada por médicos, paramédico y enfermeras(os), sobretodo en estas últimas (35). Contrastando este estudio, los padres relataron sentirse agradecidos por los cuidados proporcionados por el equipo de salud, sin hacer distinción del cuidado de enfermería, destacando del equipo al cirujano que intervino quirúrgicamente a sus hijos. Cuando hablaron de la(el) enfermera(o), refirieron distinguirla principalmente por el color azul de su uniforme, y no por el cuidado ejercido. En cuanto a su rol, destacaron su labor administrativa, como la jefatura de un servicio y supervisión de sus funcionarios; también refirieron que se encarga de entregar la educación a los padres. En relación, al trato directo con ellas(os), mencionaron que la instancia de acercamiento se dio solo cuando tenían dudas. Por otro lado, la mayoría manifestó haber vivenciado una situación traumática y triste durante el tratamiento de sus hijos; resaltando la experiencia en la unidad de recuperación anestésica, relataron sentimientos de impotencia y soledad por la falta de cercanía de las(os) enfermeras(os), solo acudieron en presencia de dudas, la mayor parte del tiempo estaban con otros pacientes o en la estación de enfermería. En este servicio no identificaron claramente a la(el) enfermera(o) del resto del equipo, solo creyeron saber quién era por el color de su uniforme. En cuanto a los cuidados de enfermería, los relacionaron con la educación sobre el cuidado y tratamiento de sus hijos, y la ejecución de algunos procedimientos. Contrastando estas vivencias con lo percibido respecto a la enfermera de la Unidad de Niños Fisurados, los entrevistados reconocieron su gestión, cercanía y dedicación, destacando su buena disposición para evacuar dudas, ellos la identificaron como una referente en todo lo relacionado con la patología.

En cuanto a las expectativas sobre el cuidado de enfermería, estas son influenciadas por la información obtenida de la actividad de este profesional, reflejado en el grado de satisfacción, utilizada como un indicador en salud, ya que aporta información sobre la calidad de los cuidados percatados por los usuarios.

El cuidado se percibe en la respuesta tanto de los pacientes como de sus padres, quienes esperan un trato digno, amable y oportuno, tal como devela el estudio "Nursing in the role of health care manager" realizado por Estefo et al (36). En relación a esto, las expectativas de los padres entrevistados fueron variadas, desde quienes solo las enfocaron en la mejoría definitiva de la malformación, hasta quienes esperaban una mejor atención por parte del equipo de enfermeras(os). Coincidiendo en el segundo grupo padres cuya experiencia en el postoperatorio inmediato fue dolorosa e impactante, que según la investigación de Estefo et al, (36), dichas vivencias son consideradas por los usuarios en salud para valorar el nivel de satisfacción respecto a la atención recibida. En lo que se refiere a los cuidados de enfermería, los sentimientos fueron ambivalentes, refiriendo gratitud hacia las(os) enfermeras(os), reconociendo su dedicación hacia labores administrativas, pero destacando su actitud distante con los pacientes y con ellos mismos. 
Respecto a los cuidados e identificación del profesional enfermero, los padres expresaron gratitud por la atención, reconociendo a la enfermera(o) como la(el) profesional encargada(o) de labores administrativas, pero que no tiene mayor cercanía con los pacientes ni acompañantes, solo acuden cuando los usuarios tienen dudas relacionadas con su situación de salud-enfermedad, siempre que sea solicitado. La distinguieron del resto del equipo por el color azul de su uniforme, siendo la característica más relevante y discriminatoria para identificarla(o).

A pesar de la existencia de estudios como el de Milos o Grau $(32,30)$, donde refieren que la(el) enfermera(o) debe ver y tratar al individuo como un ser holístico, la realidad develó lo contrario, evidenciando en las narraciones que la labor de enfermería se enfoca principalmente en funciones administrativas, lejanas al paciente, sin demostrar mayor empatía por los usuarios y menos aún por sus familiares, mostrando una atención deshumanizada, destacando además la evidente invisibilidad de enfermería que pudieron percibir los padres entrevistados.

Respecto al aporte disciplinar de la investigación, corresponde a la aplicación de la teoría de los cuidados de K. Swanson quien, a través de sus cinco procesos básicos, permite reflexionar cómo el profesional enfermero está ejerciendo el cuidado de enfermería (21, 22), evidenciando cuáles son los procesos más débiles en el ejercicio de la profesión, que requieren ser intervenidos para lograr un cuidado holístico, manteniendo una atención humanizada, tan solicitada por los pacientes y sus acompañantes. De esta forma, destacan tres procesos cómo los más alterados en las vivencias expresadas por los padres entrevistados. El primer proceso es el estar con, según Swanson, se relaciona con la emocionalidad, el acompañamiento de quien se encuentra vulnerable debido a la presencia de una enfermedad $(21,22)$. De acuerdo a las narraciones se encuentra alterado ya que los padres reconocieron la falta de acompañamiento por parte de enfermería, la falencia de ser escuchadas(os) y de un trato directo hacia ellos, destacando la labor de otros profesionales y no de las(os) enfermeras(os). El segundo proceso es el hacer por, que corresponde a la capacidad de hacer por los demás lo que se haría por uno mismo, desarrollando, por ejemplo, herramientas de empatía $(21,22)$, lo cual no fue evidenciado por este grupo de padres, ellos reconocieron esta empatía, pero en otros funcionarios; hubiesen esperado un trato más amable, sensible y cercano por parte de las(os) enfermeras(os), pero no fue percibido así. Por último, el tercer proceso alterado corresponde al posibilitar, que, de acuerdo con K. Swanson, concierne en facilitar al otro el paso hacia sucesos desconocidos $(21,22)$, en esta investigación se relaciona con la malformación labio palatina, la preparación que debieron recibir en cada una de las etapas del tratamiento desde el momento que fueron diagnosticados. Si bien algunas madres recibieron información de la enfermedad por parte de la enfermera de la Unidad de Niños Fisurados, el resto de los procesos vivenciados no fueron explicados con antelación, no se evidenció una preparación previa a la cirugía o a la recuperación postoperatoria, lo cual tal vez habría ayudado a afrontar de mejor manera la experiencia a la cual fueron sometidas(os).

Para finalizar, la investigación se basó en la teoría fenomenológica de E. Husserl, pretendiendo rescatar y develar las vivencias de los padres, cuyo aspecto en común fue el diagnóstico de sus hijos; logrando describir las experiencias tal como fueron narradas, complementadas con la emocionalidad propia del ser humano al momento de referirse a sucesos dolorosos, permitiendo comprender y evidenciar la esencia de lo descrito. 


\section{Conclusiones}

Se concluye que la de presencia una malformación labio palatina en un hijo es siempre una experiencia traumática, independiente de la etapa de la vida en que se encuentre el niño, lo cual se puede reflejar en los distintos relatos obtenidos en la investigación; destacando la presencia de sentimientos y emociones negativas, presentes también durante el proceso quirúrgico al cual es sometido este tipo de paciente.

Por otro lado, en lo que respecta a la atención recibida, los padres agradecen en general los cuidados recibidos por el equipo de salud, sin resaltar la atención de enfermería, ya que a ellas(os) las(os) identifican por su función administrativa dentro de este equipo, relatando que ellas(os) no tienen mayor cercanía con los pacientes ni sus acompañantes, solo se acercan cuando los usuarios tienen dudas relacionadas con su situación de salud-enfermedad, siempre que se les solicite. Identifican al profesional enfermero como un funcionario lejano, poco empático, que proporciona una atención deshumanizada a los usuarios y su familia, reconociéndolo en el equipo principalmente por el color azul de su uniforme.

Finalmente, la investigación pretende aportar a la profesión de enfermería respecto a los cuidados que se le entregan a los usuarios, demostrando, a través de un grupo de padres, la importancia de tener más cercanía con los pacientes, proporcionando cuidados más cálidos y humanizados, ya que no solo los procedimientos técnicos son importantes en su quehacer.

\section{Referencias bibliográficas}

1. Vanz A, Ritter N. Listening to the mothers of individuals with oral fissures. Rev esc enferm USP [Internet]. 2011 [consultado el 18 de mayo de 2018]; 45(3): 596-602. Disponible en: http://www.scielo.br/scielo.php?script=sci_arttext\&pid=S0080$62342011000300007 \& 1 \mathrm{ng}=\mathrm{en} \& \mathrm{nrm}=\mathrm{iso} \& \mathrm{t} \operatorname{lng}=\mathrm{en}$

2. Johansson B, Ringsberg K. Parents' experiences of having a child with cleft lip and palate. J Adv Nurs [Internet]. 2004 [consultado el 01 de mayo de 2018]; 47(2): 165173. Disponible en: https://www.ncbi.nlm.nih.gov/pubmed/15196190

3. Sischo L, Clouston S, Phillips C, Broder H. Caregiver responses to early cleft palate care: A mixed method. Approach. Health Psychol [Internet]. 2016 [consultado el 18 de mayo de 2018]; 35(5): 474-82. Disponible en: https://www.ncbi.nlm.nih.gov/pubmed/26280177

4. Ford A, Tastets M, Cáceres A. Tratamiento de la fisura Labio Palatina. Rev Med Clínica las Condes [Internet]. 2010 [consultado el 27 de abril de 2018]; 21(1): 16-25. Disponible en: https://www.sciencedirect.com/science/article/pii/S0716864010705010

5. Ministerio de Salud. Guía Clínica AUGE Fisura Labiopalatina [Internet]. Chile: MINSAL Gobierno de Chile; 2015, [consultado el 31 de mayo de 2018]. Disponible en: $\quad$ http://www.repositoriodigital.minsal.cl/bitstream/handle/2015/509/guiaFisuraLabioPalatina-2015-CM.pdf?sequence $=1 \&$ isAllowed=y 
6. Gómez Carretero P, Monsalve V, Soriano J, De Andrés J. Alteraciones emocionales y necesidades psicológicas de pacientes en una Unidad de Cuidados Intensivos. Med. Intensiva [Internet]. 2007 [consultado el 04 de enero de 2018]; 31(6): 318-325. Disponible en: http://scielo.isciii.es/scielo.php?script=sci_arttext\&pid=S0210$56912007000600006 \& \operatorname{lng}=\mathrm{es}$

7. Alonso López J. Programa de Preparación Psicológica en Cirugía Infantil Programada. Revista Profesional Española de Terapia Cognitivo-Conductual [Internet]. 2005 [consultado el 29 de septiembre de 2017]; 3: 56-70. Disponible en: http://files.sld.cu/anestesiologia/files/2012/08/programa-de-pre-preop-psicologicaninos.pdf

8. Sartori J, Espinoza P, Díaz M, Ferdinand C, Lacassie H, González A. ¿Qué información preoperatoria desean los padres de niños que serán operados? Rev Chil Pediatr [Internet]. 2015 [consultado el 17 de septiembre de 2017]; 86(6). Disponible en:http://www.scielo.cl/scielo.php?script=sci_arttext\&pid=S0370-

41062015000600004

9. Bellido Vallejos J, Pereira Becerra F, Cruz Hervás J. Planificación de cuidados en el paciente quirúrgico. Inquietudes [Internet]. 2007 [consultado el 30 de diciembre de 2017]; (36): 4-16. Disponible en: http://www.indexf.com/inquietudes/36pdf/36_aticulo_4.pdf

10. Universidad Virtual Manuel Fajardo [Internet]. La Habana: Facultad de Ciencias Médicas Manuel Fajardo; 2006 [consultado el 22 de noviembre de 2017]. Disponible en: http://uvsfajardo.sld.cu/unidad-vii-acto-quirurgico

11. Ramos Frausto V, Rico Venegas R, Martínez P. Percepción del familiar acompañante con respecto al cuidado de enfermería en hospitalización. Enferm glob [Internet]. 2012 [consultado el 22 de marzo de 2018]; 11(25): 219-232. Disponible en: http://dx.doi.org/10.4321/S1695-61412012000100013

12. Ministerio de Salud. Estrategia Nacional de Salud para el cumplimiento de los Objetivos Sanitarios de la Década 2011-2020 [Internet]. Santiago de Chile: Gobierno de Chile; 2018, [consultado el 04 de abril de 2018]. Disponible en: http://www.salude.cl/wpcontent/uploads/2013/08/Objetivos+Sanitarios+de+la+d\%C3\%A9\%C2\%81cada+201 1-2020.pdf

13. Rodríguez G, Gil J, García E. Metodología de la investigación cualitativa. $2^{\mathrm{a}}$ ed. Málaga: Ediciones Aljibe; 1999.

14. Sandoval CC. Perspectivas comprensivas y explicativas de la investigación social cualitativa: fundamentos teóricos y características específicas. En: Sandoval CC. Especialización en teoría, métodos y técnicas de investigación social. Investigación cualitativa. $1^{\text {a }}$ ed. Bogotá: ARFO; 2002, p. 57-59. 
15. Hungler B, Polit D. Investigación científica en ciencias de la salud. Metodología de la investigación. $6^{\text {a }}$ ed. México D.F.: McGraw Hill/ Interamericana de México; 2000. p. $2-10$

16. Soto Nuñez C, Vargas Celis I. La fenomenología de Husserl y Heidegger. Cultura de los cuidados [Internet]. 2017 [consultado el 14 de mayo de 2018]; 21(48): 43-50. Disponible en: https://rua.ua.es/dspace/bitstream/10045/69271/1/CultCuid_48_05.pdf

17. Lambert C. Edmund Husserl: la idea de la fenomenología. Teología y vida [Internet]. 2006 [consultado el 14 de mayo de 2018]; 47(4): 517-529. Disponible en: https://scielo.conicyt.cl/scielo.php?script=sci_arttext\&pid=S0049-

34492006000300008

18. Corona Fernández J. Husserl. Elementos de autorreflexión filosófica [Internet]. 2012 [consultado el 09 de junio de 2018]; 5(9): 59-90. Disponible en: http://www.scielo.org.mx/scielo.php?script=sci_arttext\&pid=S200725382012000100003

19. De Souza Minayo M. Los conceptos estructurantes de la investigación cualitativa. Salud colectiva [Internet]. 2010 [consultad el 10 de junio de 2018]; 6(3): 251-261. Disponible en: http://www.scielo.org.ar/scielo.php?script=sci_arttext\&pid=S185182652010000300002

20. Treviño Montemayor R. Actualidad de la fenomenología en psicología. Diversitas: Perspectivas en Psicología [Internet]. 2007 [consultado el 10 de junio de 2018]; 3(2): 249-261.

Disponible

en: http://www.scielo.org.co/scielo.php?script=sci_arttext\&pid=S179499982007000200007

21. Rodríguez Campo V, Valenzuela Suazo S. Swanson's care theory and her fundamental ideas, a mid-range theory for professional nursing in Chile. Enferm glob [Internet]. 2012 [consultado el 20 de mayo de 2018]; 11(28): 316-322. Disponible en: http://scielo.isciii.es/scielo.php?pid=S1695-

61412012000400016\&script=sci_arttext\&tlng=en

22. Wojnar DM. Teoría de los Cuidados. En: Raile M, editor. Modelos y teorías en Enfermería. $8^{\text {a }}$ ed. Barcelona: Elsevier; 2015, p. 670-683.

23. Hernández R, Fernández C, Baptista M. Metodología de la investigación. 5a ed. México: McGraw-Hill; 2010.

24. Rodríguez García M, Medina Moya J. Entre la complejidad y el arte: el análisis de datos en cualitativa. Index Enferm [Internet]. 2014 [consultado el 24 de marzo de 2018]; 23(3): 157-161. Disponible en: http://scielo.isciii.es/scielo.php?script=sci_arttext\&pid=S1132-12962014000200009

25. Rodríguez Yunta E. Comités de evaluación ética y científica para la investigación en seres humanos y las pautas cioms 2002. Acta bioeth [Internet]. 2004 [consultado el 04 
de diciembre de 2017]; 10(1): 37-48. Disponible en: http://www.scielo.cl/scielo.php?script=sci_arttext\&pid=S1726-569X2004000100005

26. Cornejo M. Rigor y calidad metodológica: un reto a la investigación social cualitativa. Psicoperspectivas [Internet]. 2011 [consultado el 05 de diciembre de 2017]; 10(2): 1234. Disponible en: http://www.scielo.cl/pdf/psicop/v10n2/art02.pdf

27. Jóvenes y memoria. Recordando para el futuro. Cpm: comisión provincial por la memoria [Internet]. Buenos Aires: Cpm. 2002 [consultado el 28 de enero de 2019]. Disponible

en: http://www.comisionporlamemoria.org/archivos/jovenesymemoria/bibliografia_web/ historia/Kauffman.pdf

28. Badillo Prudencio L, Diaz Urbina A, Inca Serna M, Bolaños Gil H. Estrategias de afrontamiento a las reacciones emocionales de los padres de niños con fisura labial, palatina y labiopalatina. Rev Enferm Herediana [Internet]. 2013 [consultado el 13 de diciembre de 2019]; 6(1): 12-17. Disponible en: https://revistas.upch.edu.pe/index.php/RENH/article/view/2144

29. Lafaurie M, Barón L, León D, Martínez P, Molina D, Rodríguez D, et al. Madres cuidadoras de niños(as) con cáncer: vivencias, percepciones y necesidades. Revista Colombiana de Enfermería [Internet]. 2016 [consultado el 30 de enero de 2020]; 5(5): 41-52.

Disponible

en: https://revistas.unbosque.edu.co/index.php/RCE/article/view/1423

30. Grau C, Espada M. Percepciones de los padres de niños enfermos de cáncer sobre los cambios en las relaciones familiares. Psicooncología [Internet]. 2012 [consultado el 30 de enero de 2020); 9 (1): 125-136. Disponible en: https://revistas.ucm.es/index.php/PSIC/article/view/39142

31. Casabona Martínez I, Lillo Crespo M. Gestión de cuidados de salud y competencia cultural. Cultura de los Cuidados [Internet]. 2006 [consultado el 26 de enero de 2020]; $20 \quad(1): \quad 83 . \quad$ Disponible en: https://rua.ua.es/dspace/bitstream/10045/868/1/culturacuidados_20_11.pdf

32. Milos Hurtado P, Bórquez Polloni B, Larraín Sundt A. La gestión del cuidado en la legislación chilena (II) estado actual. Ciencia y enfermería [Internet]. 2011 [consultado el 01 de junio de 2018]; 17(3): 23-33. Disponible en: https://scielo.conicyt.cl/pdf/cienf/v17n3/art03.pdf

33. Grau C, Fernández Hawrylak M. Familia y enfermedad crónica pediátrica. Anales Sis San Navarra [Internet]. 2010 [consultado el 18 de enero de 2018]; 33(2): 203-212. Disponible en: http://scielo.isciii.es/scielo.php?script=sci_arttext\&pid=S1137$66272010000300008 \& \operatorname{lng}=\mathrm{es}$

34. Ortiz Silva C, Tobar Pérez P. Desarrollando el contexto relacional y vincular: la importancia de la regulación y el vínculo materno en un preadolescente con malformación labiopalatina. Revista Clínica y Psicosocial [Internet]. 2018 [consultado el 30 de enero de 2020]; 3(3): 58-72. Disponible en: 
http://www.humanizasantiago.cl/wp-content/uploads/2019/02/Ortiz-yTobar.58a72.pdf

35. Suárez Obando F, Ordóñez A, Macheta M. Percepción de las madres con hijos afectados por malformaciones congénitas mayores: necesidad de desarrollo de un sistema de atención apropiado. Colombia Médica [Internet]. 2009 [consultado el 30 de enero de 2020]; 40(1): 85-94. Disponible en: https://www.redalyc.org/pdf/283/28340111.pdf

36. Estefo Agüero S, Paravic Klijn T. Nursing in the role of health care manager. Cienc enferm [Internet]. 2010 [consultado el 27 de enero de 2020]; 16(3): 33-39. Disponible en: https://scielo.conicyt.cl/scielo.php?pid=S071795532010000300005\&script=sci_arttext\&tlng=en

Cómo citar: San Martín Osses V, Osorio Spuler X. Vivencias de padres de niños con fisura labio palatina respecto al cuidado en las distintas etapas del tratamiento quirúrgico. Enfermería: Cuidados Humanizados. 2021; 10(1): 44-59. Doi: https://doi.org/10.22235/ech.v10i1.2411

Contribución de los autores: a) Concepción y diseño del trabajo, b) Adquisición de datos, c) Análisis e interpretación de datos, d) Redacción del manuscrito, e) Revisión crítica del manuscrito. V. S. M. O. ha contribuido en a, b, c, d, e; y X. O. S. ha contribuido en a, b, c, d, e.

Editora científica responsable: Dra. Natalie Figueredo. 